\title{
Implementing Hierarchical Indoor Semantic Location Identity Classification: A Case Study for COVID-19 Proximity Tracking in the Philippines
}

\author{
Irvin Kean Paulus PADERES ${ }^{\text {a }}$, Ligayah Leah FIGUEROA ${ }^{a}$ and Rommel FERIA ${ }^{a}$ \\ ${ }^{a}$ University of the Philippines - Diliman, Quezon City, Philippines
}

\begin{abstract}
Efforts toward COVID-19 proximity tracking in closed environments focus on efficient proximity identification by combining it with indoor localization theory for location activity monitoring and proximity detection. But these are met with concerns based on existing considerations of the localization theory like costly infrastructure, multi-story support, and over-reliance on sensor networks. Semantic location identities (SLI), or location data stored with additional meaningful context, has become a feasible localizing factor especially in locations that have multiple spaces with different usage from each other. There is also a novel method of classification framework, called hierarchical classification, that leverages the hierarchical structure of the labels to reduce model complexity. The research aims to provide a solution to proximity analysis and location activity monitoring considering guidelines released in a Philippine context that addresses concerns of indoor localization and handling of geospatial data by implementing a hybrid hierarchical indoor semantic location identity classification that focuses on observable events within context-unique locations.
\end{abstract}

Keywords. indoor localization, fingerprint localization, machine learning, classification problem, hierarchical classification, semantic location identities

\section{Introduction}

In the interest of public health due to the worldwide COVID-19 disease outbreak, the easing of community quarantines has been slow and steady for most countries. Governments enforced guidelines maintaining public health and social measures (PHSM) to continue mitigating the spread of the disease. Commonly, these countries have been collecting appropriate surveillance data in order to track public health indicators to allow proper authorities to reassure stakeholders that it is now safe to go back to the physical premises. Location-based solutions (LBS) [4] are one of the most commonly implemented with focus on contact tracing and proximity tracking, with most of the solutions implemented focus on contact tracing. Proximity tracking, on the other hand, is much harder to implement mainly because of the implementation barrier where it is tied to licensed software solutions. In the hopes of easing this barrier, the use of machine learning has been a good way to optimize received signal strength indicator (RSSI) from sensor 
nodes measurements for fingerprint-based localization [14]. The normal use-case of indoor localization is based on the estimation of precise location of the user over an indoor space, but in the context of proximity tracking, there is only a need to track the location usage within the sensing area. Machine learning (ML) classifiers do the job of estimating the user's location based on fingerprint data, but most research tends to ignore a latent aspect of localization, which is location data context. This research aims to introduce the use of semantic location identities (SLI) in order to utilize semantic ontology [10] and group fingerprint data into meaningful areas such as bathrooms, office rooms, hallways, etc., in order to reduce the localization model's learning complexity. On the other hand, most work on localization systems using ML algorithms [2] only focuses on using a traditional flat classification framework where the predicted labels have the same level with each other. This works for fingerprint-based localization, but as shown by SLIs, the context of any location has a natural hierarchy governing it in the form of buildings, floors, and the user's location. There is another type of framework that has the capability to utilize this hierarchical design of location data called hierarchical classification [12]. This classification framework accepts and utilizes the hierarchical relationships of each output label in order to utilize this inherent design by utilizing different local classifiers for each hierarchical class. Moving forward with this concept, one could leverage the use of cluster analysis on fingerprint data to identify similarities and extract groupings out of it that can be utilized as another hierarchical class. Zhang et al. [17] explored the use of cluster algorithms in providing another layer of hierarchy to the location data. Their work was able to show that introducing partitions that allow overlapping membership was able to improve the localization performance of hierarchical classifiers by allowing better stability and generalization of the input data. With this in mind, exploring other clustering algorithms that allow overlapping memberships can also be explored to improve the classification framework. This research aims to explore the performance of these hierarchically-structured local classifiers, in which each label type node has its own local classifier and the nodes have a parent-child relationship, in providing the final prediction of the system.

In order for proximity tracking to be an attractive COVID-19 mitigation solution to consider, there is a need to improve its performance within indoor spaces. Current solutions do not reliably maintain public health measures unobtrusively within indoor facilities without the use of proprietary software and equipment. The purpose of this research is to leverage the hierarchical structure of location data in improving fingerprint localization by the use of hierarchical classifier framework and integrate both SLI, identities focusing on observable events within context-unique locations, and area partitions, focused on clustering fingerprint data as part of the SLIs, as integral parts of the hierarchical classification. The goal of this hybrid method is to decrease the complexity of the estimation models and bring improvement to the performance of proximity tracking systems in indoor locations. In this regard, the objectives of the research will be focused on the following:

1. Improve the area partitioning performance of the framework by exploring the use of clustering algorithms that allow overlapping membership,

2. Determine an optimal configuration of local classifiers by exploring different machine learning algorithms for each hierarchical class, i.e floor classification, cluster classification, and SLI classification 
3. Leverage the hierarchical structure of location data with the innate cluster capabilities of semantic location identities into a hybrid hierarchical SLI classifier

\section{Literature Review}

Early work towards the use of hierarchy in order to improve fingerprint localization exists, albeit in different forms. The work of Kim et al. [7] was able to utilize deep neural networks (DNN) in order to integrate hierarchy into localization. They were able to reduce learning complexity and have favorable results although the composition of DNN brings forth the consideration of the added time and technological complexity required in order to train and predict in DNNs. On the other hand, Seçkin and Coşkun [11] shows a different approach to tackling hierarchical improvement to fingerprint localization by iteratively training and predicting over different hierarchies of location data (building, floor, then honing in to the longitudinal and latitudinal position). The study showed favorable improvements in classifying between the parent and sub-parent classes (e.g. building to floor), but its performance on estimating the latitudinal and longitudinal values of the user's location failed to present significant improvements compared to traditional algorithms. The work of Zhang et al. [17] tackled the use of hierarchical classification framework for localization, with the authors exploring area partitioning by grouping fingerprint data with the use of clustering algorithms. They introduced overlapping membership into the cluster algorithm, with them utilizing a modified K-Means Clustering algorithm that allows membership overlap, and found that it doesn't affect the predictive performance of the model. In reality, it improved its generalization capabilities of the framework by having more RSSI samples to match zones and allow more statistical information to be utilized. Though the authors were still able to show the improvement of overlapping clusters, the use of other clustering algorithms that allow overlapping data can still be explored to bring significant improvement to the performance of hierarchical classification.

This research hopes to explore two methods to create a hybrid hierarchical classification framework, one is the exploration of different clustering algorithms that have overlapping membership built into it, and the other focuses on utilizing SLI as the final hierarchical class to be classified. Zhang et al. [17] showed great performance with their improved k-means algorithm, but it failed to have a better performance compared to Fuzzy C-Means (FCM) which natively allows membership overlap. For this research, the authors aim to explore a version of DBSCAN called HDBSCAN [3]. The algorithm has yet to be explored in its performance for localization, but as shown by the study of Malzer and Baum [9], the hierarchical tree structure was able to show data points that has the same membership to a parent tree and it gave a high cluster validation measure of perfect Adjusted Rand Index (ARI) score and zero false noise classification rate in clustering GPS datasets. In this research, the author aims to utilize the innate use of hierarchy of HDBSCAN in zone cluster selection. Another substantial change that will be implemented to hierarchical classification is the use of semantic location identities as a classification factor of the final hierarchical level, the position classifier. Based on the study of [16], the fusion of SLI information in a sensing layer can not only provide a great context model engine for LBS applications but it can also provide enrichment of location identification of its system. The work of Abdelnasser et al. [1] showed its ef- 
fectiveness in improving localization models in an indoor context. The study used landmarks based on sensor fusion and localization techniques to improve semantic localization and mapping (SLAM) model performance. Even if the study used dead-reckoning instead of fingerprinting, the process of capturing historical values to formulate semantic landmarks can be used to improve localization. Van Woensel et al. [15] implemented the use of SLI-improved fingerprinting as a verification model for the estimated position computed by a ML model based on C.50 Decision Trees that uses fingerprint data within the sensed area. The performance of the localization model with semantic location verification was tested compared to the utilization of a ML model alone and results from the evaluation show an increase in accuracy $(92.8 \%)$ of the semantic location model in a flat classification framework.

For the localization models, the composition of the algorithms we compare as possible classifiers to the hybrid framework came from their previous performances as flat classifiers. In the study of [17], they utilized a simple 1-Nearest Neighbor algorithm, a special case of K-NN that classifies objects using a voting mechanism and selects the most voted class among the 1- nearest neighbors closest to the object. This relatively simple algorithm is appropriate enough for floor and area partition classification, but there are other algorithms that can be utilized that don't rely on explicit computations but have the possibility to perform better. Locality Sensitive Hashing (LSH) [13] is an approximate algorithm, given that it is able to provide a good approximation of the final estimate which can be used to identify neighbor membership. From the findings of Koga et al. [8], LSH's performance shows more than 0.94 cophenetic correlation coefficient (CCC) which means it has a high performance in detecting closely linked clusters while maintaining low complexity, which can be approximated to detect which cluster an input belongs to. The work of Seçkin and Coşkun [11] explored the use of Random Forest algorithm (RF) which is an ensemble of decision trees that has a random selection of the total features of the training dataset and the mode value from all the trees is used as output. In floor identification alone, this algorithm was able to acquire $95 \%$ classification accuracy in a building that was reinforced by prior training on a fingerprint classifier which can be utilized for floor and area partition classification, as well as SLI classification due to its reliance on training. The algorithms chosen for SLI classifiers should be more complex than the previous two hierarchical classes as it signifies the final position to be localized. Zhang et al. [17] found significant performance gains in location classification accuracy with the use of Support Vector Machines (SVM). It is an algorithm originally designed for binary classification problems, but a one-versus-one heuristic method can be used that splits the dataset into binary problems with the use of $N(N-1) / 2$ binary SVM classifiers and the class from the multi-label setup with the most predictions or votes is selected to be the final output. Zhang et al. showed a $64.97 \%$ accuracy of the SVM+KNN hierarchical classifier in position classification, but this experiment was not utilizing SLI as the last hierarchical class and this will be explored in this research. This research will also use Random Forest and C5.0 Decision Trees as discussed previously. The last three models chosen were part of the algorithms discussed in the survey of [18], but performance measurements in terms of real-life localization classification were not disclosed. Extreme learning machines and radial basis function neural networks, which are feedforward neural networks that have the characteristics of close network structure and rapid learning that eliminates the problem of excessive training time. And the last algorithm, Adaptive Boosting (AdaBoost), is another ensemble algorithm that combines 
Table 1. Initial Results of the Algorithms to be Explored for the Hybrid Classifier Framework Based on Existing Literature

\begin{tabular}{|c|c|c|}
\hline Algorithm & Performance & Notes \\
\hline \multicolumn{3}{|c|}{ Area Partitioning Algorithms } \\
\hline $\begin{array}{l}\text { Modified K-Means Algorithm } \\
\text { with Overlap [17] }\end{array}$ & Matched actual reference points numbers & $\begin{array}{l}\text { Dependent on } p \text { threshold value to determine } \\
\text { number of clusters }\end{array}$ \\
\hline $\begin{array}{l}\text { Fuzzy C-Means (FCM) } \\
\text { Clustering [17] }\end{array}$ & $\begin{array}{l}\text { Natively allows overlap on clusters due } \\
\text { to arbitrary } p \text { values, }\end{array}$ & \\
\hline HDBSCAN [3] & $\begin{array}{l}\text { Good AUC showing promising clustering } \\
\text { in terms of data features }\end{array}$ & Also allow native class cluster overlap \\
\hline \multicolumn{3}{|c|}{ Zone Classifier Algorithms } \\
\hline 1-Nearest Neighbor [17] & $2.25 \%$ zone classification rate & Provides fast and easy zone classification \\
\hline $\begin{array}{l}\text { Locality Sensitive } \\
\text { Hashing }[8]\end{array}$ & $94 \% \mathrm{CCC}$ & $\begin{array}{l}\text { CCC measures performance in detecting closely-linked } \\
\text { structures, meaning better determination of natural data } \\
\text { overlap into other classes }\end{array}$ \\
\hline Random Forest [11] & $95 \%$ accuracy & High performance in general classification \\
\hline \multicolumn{3}{|c|}{ SLI Classifier Algorithms } \\
\hline $\begin{array}{l}\text { Support Vector } \\
\text { Machines (SVM) [17] }\end{array}$ & $64.97 \%$ accuracy & $\begin{array}{l}\text { Tests here classify for actual position that is why it has } \\
\text { lower accuracy compared to other algorithms }\end{array}$ \\
\hline Random Forest [11] & $95 \%$ accuracy & $\begin{array}{l}\text { Classifies for floor position which is more general than } \\
\text { position and SLI values }\end{array}$ \\
\hline C5.0 Algorithm [15] & $92.8 \%$ accuracy & The only test that utilizes SLIs for indoor classification \\
\hline $\begin{array}{l}\text { Extreme Learning } \\
\text { Machines (ELM) [18] }\end{array}$ & $<$ No test results $>$ & \multirow{3}{*}{$\begin{array}{l}\text { These algorithms were recommended by the survey of } \\
\text { Zhu et al. [18] based on their performance. }\end{array}$} \\
\hline $\begin{array}{l}\text { Radial Basis Function } \\
\text { Neural Network (RBFNN) [18] }\end{array}$ & $<$ No test results $>$ & \\
\hline $\begin{array}{l}\text { Adaptive Boosting } \\
\text { (AdaBoost) [18] }\end{array}$ & $<$ No test results $>$ & \\
\hline
\end{tabular}

several weak classifiers with their outputs put into a voting mechanism in order to provide a simple and fast improvement to the base classification capabilities of the weak model. A brief summary of the algorithms to be explored is found in Table 1. These algorithms were chosen to implement a hybrid localization framework that integrates hierarchical classification and semantic location identity together. The research also aims to achieve localization without provisioning costly infrastructure with the efficient use of sensor nodes.

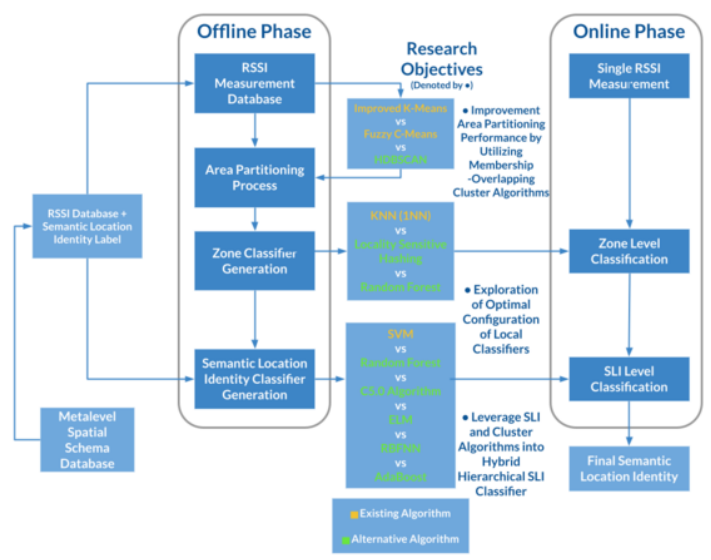

Figure 1. Hybrid Localization Process and Research Workflow Diagram

\section{Methodology}

Based on the defined objectives in Section 1, the proposed research focuses on providing an improved localization framework that will extend IPS by processing sensed location 
from various sensor data in a hierarchical classifier that systematically estimates the position within the sensed area from a general class to a specific SLI location. The evaluation method for the objectives will also be discussed in this section.

\subsection{Proposed Experimental Design}

Figure 1 visualizes the proposed localization framework and the direction of the research as a whole. The main localization process follows the principle of divide-and-conquer, separating the focus between classifying the general classes (i.e. floors and area clusters), and classifying the SLI as the current location of the user. Significant focus will also be given to the area clustering algorithm as related literature shows the correlation of the clustering algorithm to the performance of the cluster classifiers. In training the classifiers, a 6040 split will be conducted in order to prevent the machine learning algorithms from overfitting into the fingerprint dataset. In this split, $60 \%$ of the dataset will be for training and testing, while the latter $40 \%$ is for evaluation. The data belonging to the $60 \%$ split will also be subjected to a $k$-fold cross-validation procedure which will rotate the data between training and testing dataset. This procedure divides the observations into $k$ groups of approximately equal size where the observations on the model will come from the mean of the $k$ group scores and their variance.

\subsection{Improvement of the Framework's Area Partitioning Performance}

For this objective the research proposes the comparison of previously utilized cluster algorithms to other algorithms that allows membership overlap between area partitions. The final output of this objective will be the final clusters to be used by the proposed framework. One thing to note is that the creation of the area clusters with each of the area clusters is done to the whole fingerprint dataset before the initial 6040 split and $\mathrm{k}$-fold cross validation as the area clusters are required by the proposed framework as a hierarchical class. The evaluation of the area partition classifiers will also act as the evaluation of this objective due to the direct effect of these cluster algorithms to this hierarchical class.

\subsection{Determining the Optimal Configuration of Local Classifiers}

The total localization performance of the hybrid framework will really depend on the classification models used for each hierarchical class, and because of this a sufficient amount of effort will be conducted in finding the final set of classifiers. The hierarchical classification framework starts with the classification of the floor where the RSSI was captured. In terms of the hierarchical levels, the classification difficulty at this first level is relatively low. The algorithms to be evaluated will be the same for both hierarchical classes. One thing to consider for area partition classification is that the presence of overlap over zones reduces the need for classification models for pre-training. SLI classification, on the other hand, carries the bulk of the complexity of the localization process. Even with the reduced scope due to floor and cluster classification, classifiers in this hierarchical class require a more robust training. In order to verify the performance of the local classifier algorithms without worrying about overfitting to the split $60 \%$ data, a cross-validation process will be conducted to get the training and testing data to be used for evaluating the local classifier algorithms. The validation process loops over differ- 
ent "folds" of the dataset with different training and testing subsets for each loop. This process allows the study to measure the real-world performance of the algorithms when classifying data by simulating scenarios where the model classifies data that it is not trained on.

For each loop within the cross-validation process, these measurements are taken:

- Accuracy - the most intuitive performance measure, ratio of correct predictions over the total number of observations

- Precision - ratio of correct positive predictions over total predicted positive observations, which are both the true and false positives

- Recall - checks the sensitivity of the prediction, the ratio of correct positive predictions over the total observations in the class to be predicted, which are the true positive and false negatives

- F1 Score - the weighted average of the Precision and Recall

After all of the loop is finished, the mean of these metrics will be taken as the final value of the classifier configuration. In order to measure the spread between the measurements in order to determine if the performance of the models for each loop varies, the Standard Deviation is taken. It measures the dispersion of the score values from the mean of the scores from all of the loop.

\subsection{Hybrid Hierarchical SLI Classifier}

Hierarchical classification framework follows the well-known divide-and-conquer (DAC) principle, with the aim to reduce the input space of different general subspaces in order to improve the framework's generalization capability as well as its learning performance. The use of location data hierarchy will reduce the complexity of the proximity tracing problem by having a smaller number of SLI to consider in classification. This change will still be applicable in the context of proximity tracing problem, by defining the area of the identity as metadata, the system can determine if the number of users to localize the same SLI reaches the approximate population density threshold that will break the enforced social distancing rule of $\mathbf{1}$ meter as defined by the guidelines set by Philippine government institutions such as CHE and CSC $[6,5]$. To help define these identities and their metadata, a metalevel spatial schema showing the relationship of static and dynamic elements of the sensed area will be formulated. The output framework will utilize fingerprint values based on all RSSI values captured from the Bluetooth sensor network. For evaluating the classification performance of the hierarchy-improved algorithms in each class of the data hierarchy, their performance will be compared to base algorithms in order to show if hierarchical classifiers provide significant improvement against flat classifiers. The comparison of the total running time of the different classifier configuration will be taken in order to check how the training and prediction time of the hierarchical classification framework compares to the flat classification framework. After determining the optimal configuration for the hierarchical classification framework, its performance will be tested on the remaining $40 \%$ data to add another verification on the performance of the model in classifying new data. The confusion matrix for each hierarchical class of this configuration will also be used to determine its performance in classifying the class labels for each hierarchy. 


\section{Results and Discussion}

This research is an ongoing study that just passed its approval phase. But as defined in the previous section, most of the framework has been clearly defined based on existing results of other studies related to it. For this section, the work towards the proposed experimental design will be discussed, specifically on the contemporary algorithms that the research will explore in order to define what this study aims to bring into hierarchical classification implementation that differentiates it from existing studies.

\subsection{Improvement of the Framework's Area Partitioning Performance}

This research aims to explore the performance of HDBSCAN, an extended version of DBSCAN which is a density-based clustering algorithm where it now integrates hierarchy in order to get clusters, in improving the zone classification capabilities of the whole framework. It was chosen based on its ability to define a distance threshold parameter that defines the radius size and minimum population of points in order to control membership between clusters, allowing for overlapping membership. HDBSCAN is generally a transductive method where it uses new data points to alter the underlying clustering within its trained dataset. But for all intents and purposes of this research there is not a need to redefine the generated clusters as future RSSI captures are, by design, recaptured data within the localized area. This algorithm will be compared against previously utilized cluster algorithms such as modified K-Means and Fuzzy C-Means. K-means will be implemented here to utilize centroids of the RSSI measurements to assign them into clusters and group them based on a threshold value. Fuzzy C-Means will be implemented in this research as is due to it natively computing the degree of membership of data to a cluster. In previous works, the RSSI measurements are collected by the reference point (RP) where RPs are the one to be computed as to which area partition they belong to. But in order to fit this study, in the purposes of experimentation, there will be a switch from the use of RPs into semantic location identities. This change will still fit the algorithm, as each SLI will be a vector of RSSI measurements at different RP.

\subsection{Determining the Optimal Configuration of Local Classifiers}

For floor and area partition classifiers, the research introduces the following algorithms, Locality Sensitive Hashing and Random Forest, to be compared to the 1-Nearest Neighbor algorithm. The main idea behind the use of LSH is its low complexity just like 1NN. Given that it is an approximate algorithm, the research aims to leverage the algorithm's composition and see if it will bring significant improvement for classification accuracy, if not, the training time itself. Random forest, on the other hand, was chosen based on its previous performance on floor classification. Given that both floor and area partition classes have more generality, the RF algorithm can be sufficiently used for this use-case.

The algorithms chosen for SLI classifiers have more complexity than the previous ones for floor and area partitions. This study will use Support Vector Machines as a baseline SLI classifier for comparison to the following algorithms to be explored:

- Random Forest - the structure of the Random Forest to be utilized here is similar to the one used for area cluster and floor classification. Given that SLI is the last class to be classified, the study aims to leverage the use of all the fingerprint data features for prediction 
- C5.0 Algorithm - C5.0 is an offshoot of the decision tree algorithm that uses less memory and a smaller rule set but it is able to achieve high accuracy. The available features used for this study are compact enough for the C5.0 algorithm to work well

- Extreme Learning Machines - an algorithm based on feedforward neural networks, the difference is that the hidden nodes are randomly assigned and it doesn't need to be tuned. The great thing behind this algorithm is its ability to be trained in a single step, essentially amounting to learning a linear model

- Radial Basis Function NN - also based on neural networks, the difference being that it uses radial basis function as the activation function. RBF allows neural networks to utilize the rate of closeness of a data point to its label for prediction

- AdaBoost - for this research, the AdaBoost algorithm will use Random Forest as the weak classifier. This study aims to utilize the performance of RF in an ensemble form to improve its performance

\section{Conclusion}

Based on Table 1, initial results from related literature shows the feasibility of these changes in providing improvements in the hybrid classification framework. The main purpose of this framework is to use it for a proximity tracking solution to provide localization capabilities to a system that is not too complicated but can bring comparative performance with lower sensor topology. The research also aims to show the feasibility of this mitigation solution as a response to COVID-19. Prior solutions show as well how LBS can utilize proximity tracking in maintaining health guidelines for COVID-19. This improvement is aimed for educational institutions and office agencies to provide reliable solutions for maintaining public health and social measures and help provide solutions should the need to go back to commercial and educational spaces arise. Most of the limitations of the study were due to the current quarantine situation affecting how the research will conduct the experiments. This study will not delve in exploring the performance of the localization model against device heterogeneity due to lack of sufficient sample devices. Also, this study will also not explore security concerns of crowdedness detection systems and the possible vulnerabilities that sensor-dependent systems. Future work for this study is the main output, a novel hierarchical localization framework revolving around comparison between the use of relative positioning with SLI and estimation of exact coordinates in a given space and the assembly of the hierarchical classification system based on the fusion of the optimal local learning models acquired through experimentation.

\section{Acknowledgements}

This research was funded by the University of the Philippines Diliman through the Office of the Vice Chancellor for Research and Development. 


\section{References}

[1] Heba Abdelnasser, Reham Mohamed, Ahmed Elgohary, Moustafa Farid Alzantot, He Wang, Souvik Sen, Romit Roy Choudhury, and Moustafa Youssef. Semanticslam: Using environment landmarks for unsupervised indoor localization. IEEE Transactions on Mobile Computing, 15(7):1770-1782, 2015.

[2] Osamah Ali Abdullah and Ikhlas Abdel-Qader. Machine learning algorithm for wireless indoor localization. In Machine Learning-Advanced Techniques and Emerging Applications. IntechOpen, 2018.

[3] Ricardo JGB Campello, Davoud Moulavi, Arthur Zimek, and Jörg Sander. Hierarchical density estimates for data clustering, visualization, and outlier detection. ACM Transactions on Knowledge Discovery from Data (TKDD), 10(1):1-51, 2015.

[4] Mohammad Jabed Morshed Chowdhury, Md Sadek Ferdous, Kamanashis Biswas, Niaz Chowdhury, and Vallipuram Muthukkumarasamy. Covid-19 contact tracing: Challenges and future directions. 2020.

[5] CSC. Revised interim guidelines for alternative work arrangements and support mechanisms for workers in the government during the period of state of national emergency due to covid-19 pandemic. http: //www.csc.gov.ph/phocadownload/MC2020/MC \\%20No. $\ \% 2010, \backslash \% 20$ s. $\backslash \% 202020$.pdf, 2020. Accessed: 2020-11-06.

[6] DepEd. Guidelines on the required health standards in basic education offices and schools. https: //www.deped.gov.ph/wp-content/uploads/2020/06/DO_s2020_014.pdf, 2020. Accessed: 202011-06.

[7] Kyeong Soo Kim, Sanghyuk Lee, and Kaizhu Huang. A scalable deep neural network architecture for multi-building and multi-floor indoor localization based on wi-fi fingerprinting. Big Data Analytics, 3(1): 1-17, 2018.

[8] Hisashi Koga, Tetsuo Ishibashi, and Toshinori Watanabe. Fast hierarchical clustering algorithm using locality-sensitive hashing. In International Conference on Discovery Science, pages 114-128. Springer, 2004.

[9] Claudia Malzer and Marcus Baum. A hybrid approach to hierarchical density-based cluster selection. In 2020 IEEE International Conference on Multisensor Fusion and Integration for Intelligent Systems (MFI), pages 223-228. IEEE, 2020.

[10] R. Rocha. Middleware for location-based services. 2004.

[11] Ahmet Çağdaş Seçkin and Aysun Coşkun. Hierarchical fusion of machine learning algorithms in indoor positioning and localization. Applied Sciences, 9(18):3665, 2019.

[12] Carlos Silla and Alex Freitas. A survey of hierarchical classification across different application domains. Data Mining and Knowledge Discovery, 22:31-72, 01 2011. doi: 10.1007/s10618-010-0175-9.

[13] Malcolm Slaney and Michael Casey. Locality-sensitive hashing for finding nearest neighbors [lecture notes]. IEEE Signal processing magazine, 25(2):128-131, 2008.

[14] Santosh Subedi and Jae-Young Pyun. Practical fingerprinting localization for indoor positioning system by using beacons. Journal of Sensors, 2017, 2017.

[15] William Van Woensel, Patrice C Roy, Syed Sibte Raza Abidi, and Samina Raza Abidi. Indoor location identification of patients for directing virtual care: An ai approach using machine learning and knowledgebased methods. Artificial Intelligence in Medicine, 108:101931, 2020.

[16] Xin Wang, Jianga Shang, Fangwen Yu, and Jinjin Yan. Indoor semantic location models for locationbased services. Int. J. Smart Home, 7(4):127-136, 2013.

[17] Chenbin Zhang, Ningning Qin, Yanbo Xue, and Le Yang. Received signal strength-based indoor localization using hierarchical classification. Sensors, 20(4):1067, 2020.

[18] Xiaoqiang Zhu, Wenyu Qu, Tie Qiu, Laiping Zhao, Mohammed Atiquzzaman, and Dapeng Oliver Wu. Indoor intelligent fingerprint-based localization: Principles, approaches and challenges. IEEE Communications Surveys \& Tutorials, 2020. 